ISSN 2590-9770

The Art of Discrete and Applied Mathematics 2 (2019) \#P1.08

https://doi.org/10.26493/2590-9770.1301.165

(Also available at http://adam-journal.eu)

\title{
On primitive geometries of rank two*
}

\author{
Dimitri Leemans ${ }^{\dagger}$ \\ Université Libre de Bruxelles, C.P.216 - Algèbre et Combinatoire, \\ Boulevard du Triomphe, 1050 Brussels, Belgium \\ Bernardo G. Rodrigues \\ School of Mathematical Sciences, University of KwaZulu-Natal, \\ Durban 4041, South Africa
}

Received 9 September 2015, accepted 18 September 2018, published online 14 August 2019

\begin{abstract}
In this paper, we describe a new algorithm to classify primitive coset geometries of rank two for a given group $G$. This algorithm allows us to classify those geometries for the 12 smallest sporadic simple groups.
\end{abstract}

Keywords: Primitive coset geometries, sporadic groups, codes, designs.

Math. Subj. Class.: 51E24, $20 D 45$

\section{Introduction}

Primitive coset geometries have been studied since the 1990's, first by a team led by Francis Buekenhout in Brussels [4, 5] and later on by Peter Rowley and Nayil Kilic (see for instance $[8,9,10,11,12])$. These geometries may be used to construct codes, designs, graphs, etc. Recently, we had the idea of using rank two primitive geometries to construct new binary codes for the McLaughlin group [13]. More precisely, we examined the binary codes obtained from the row span over $\mathbb{F}_{2}$ of the adjacency matrices of some strongly regular graphs which occur as subgraphs of the McLaughlin graph, namely those with parameters $(105,32,4,12),(120,42,8,18)$ and $(253,112,36,60)$. These new codes were obtained by computing incidence matrices of rank two geometries whose elementstabilizers are maximal subgroups of the McLaughlin group. In order to be able to generate these geometries, a new approach was needed. Indeed, the previous algorithms described by Dehon and Leemans [7] did not allow for a study of a group such as the Mathieu group

\footnotetext{
* The authors also thank an anonymous referee for useful comments that improved this paper.

$\dagger$ Corresponding author.

E-mail addresses: dleemans@ulb.ac.be (Dimitri Leemans), rodrigues@ukzn.ac.za (Bernardo G. Rodrigues)
}

(a) (i) This work is licensed under http://creativecommons.org/licenses/by/3.0/ 
$M_{24}$ of order roughly $2.4 \cdot 10^{8}$. We managed to classify such geometries with our new algorithm for the 12 smallest sporadic groups, the largest one being the Rudvalis group, of order roughly $1.4 \cdot 10^{11}$. The geometries obtained are available on the following websites:

- https://data.adam-journal.eu/apg/

- http://homepages.ulb.ac.be/ dleemans/PRI/

Our idea relies on permutation representations of groups. We present in this paper an algorithm that outperforms the previous algorithms by a factor of 1000 at least. It allows to classify rank two primitive coset geometries for the five Mathieu groups, the first three Janko groups, the Higman Sims group, the McLaughlin group, the Held group and the Rudvalis group. It also allows to confirm the classification of rank two primitive geometries of $M_{11}$ given in [5], $M_{23}$ given in [10] and to correct some infelicities in [12] for $M_{22}$. We also obtained a complete classification for $M_{24}$ while Kilic gives in [11] a partial one. The results for the Janko groups, the Higman Sims group, the McLaughlin group, the Held group and the Rudvalis groups are complete and new.

\section{Terminology}

The basic concepts about geometries constructed from a group and some of its subgroups are due to Tits [14] (see also [3, Chapter 3]). The following theorem shows how to construct geometries starting from groups.

Theorem 2.1 (Tits, 1956 [14]). Let $n$ be a positive integer. Let $I:=\{1, \ldots, n\}$ be a finite set and let $G$ be a group together with a family of subgroups $\left(G_{i}\right)_{i \in I}$. Let $X$ be the set consisting of all cosets $G_{i} g, g \in G, i \in I$. Let $t: X \rightarrow I$ be defined by $t\left(G_{i} g\right)=i$. Define an incidence relation $*$ on $X \times X$ by:

$$
G_{i} g_{1} * G_{j} g_{2} \text { iff } G_{i} g_{1} \cap G_{j} g_{2} \text { is non-empty in } G \text {. }
$$

Then the 4-tuple $\Gamma:=(X, *, t, I)$ is an incidence structure having a chamber. Moreover, the group $G$ acts by right multiplication as an automorphism group on $\Gamma$. Finally, the group $G$ is transitive on the flags of rank less than 3.

In the Theorem 2.1 above, a chamber is a set containing $n$ cosets that are pairwise nondisjoint. Let $G$ be a group and $\left\{G_{i}: i \in\{1, \ldots, n\}\right\}$ be a set of subgroups of $G$. We call $\Gamma\left(G ;\left(G_{i}\right)_{i \in I}\right)$ the coset geometry associated to $G$ and the subgroups $\left(G_{i}\right)_{i \in I}$ using the above theorem. By Theorem 2.1, we see that the group $G$ acts on $\Gamma$ as a type-preserving automorphism group.

In this paper, we only deal with geometries of rank two, that is coset geometries constructed from a group $G$ and two subgroups $G_{1}, G_{2}$ of $G$. Let us denote such a geometry $\Gamma:=\Gamma\left(G ;\left(G_{1}, G_{2}\right)\right)$. We readily have that $\Gamma$ is flag-transitive, that is, $G$ acts transitively on the pairs of non-disjoint cosets of $\Gamma$. Moreover, we want $\Gamma$ to be primitive, meaning that $G_{1}$ and $G_{2}$ must be maximal subgroups of $G$. Finally, as $G_{1}$ and $G_{2}$ are maximal subgroups of $G$, we necessarily have $\left\langle G_{1}, G_{2}\right\rangle=G$ provided $G_{1} \neq G_{2}$. This implies that $\Gamma$ is a firm and connected geometry.

We are thus interested in classifying firm, connected, flag-transitive and primitive coset geometries of rank two for a given group $G$. Given two coset geometries $\Gamma_{1}:=\Gamma(G$; $\left.\left(G_{1}, G_{2}\right)\right)$ and $\Gamma_{2}:=\Gamma\left(G ;\left(H_{1}, H_{2}\right)\right)$, we say that $\Gamma_{1}$ and $\Gamma_{2}$ are isomorphic provided there 
exists an element $g \in \operatorname{Aut}(G)$ such that $g\left(\left\{G_{1}, G_{2}\right\}\right)=\left\{H_{1}, H_{2}\right\}$. In the next section, we describe an algorithm to classify, up to isomorphism, all firm, connected, primitive geometries of rank 2 for a given group $G$.

Given a geometry $\Gamma:=\Gamma\left(G ;\left(G_{1}, G_{2}\right)\right)$, its dual $\Gamma^{*}$ is the geometry

$$
\Gamma^{*}:=\Gamma\left(G ;\left(G_{2}, G_{1}\right)\right)
$$

To a coset geometry of rank two, $\Gamma\left(G ;\left(G_{1}, G_{2}\right)\right)$ we can associate a graph called the incidence graph $\mathcal{G}$ whose vertices are the cosets of $G_{1}$ and $G_{2}$, two distinct cosets being joined by an edge provided their intersection is nonempty. This graph is bipartite and $G$ acts transitively on the edges of $\mathcal{G}$. Following [2], the gonality $g$ of $\Gamma$ is half the girth of $\mathcal{G}$. The point-diameter $d_{p}$ (resp. line-diameter $d_{l}$ ) is the largest distance from the coset $G_{1}$ (resp. $G_{2}$ ) to any other coset of $\Gamma$. To $\Gamma$, we associate the triple $\left[d_{p}, g, d_{l}\right]$. Finally, we recall that a design $S_{\lambda}(t, k, v)$ is a geometry of rank two with $v$ points and lines of size $k$ such that each set of $t$ points is contained in exactly $\lambda$ lines.

\section{Algorithm}

\subsection{The old algorithm}

The following algorithm was described in [7] (see Section 3, top-down approach) to construct residually weakly primitive geometries. It was the same algorithm that was used by Dehon in [6] to classify primitive geometries. This algorithm constructs primitive geometries of all possible ranks, not only rank two.

Sketch of the Dehon algorithm. Let $G$ be a group for which we want to compute all firm, residually connected, flag-transitive and primitive geometries. Construct a set $S$ containing all maximal subgroups of $G$ and the group $W=\operatorname{Aut}(G)$ acting as a permutation group on $S$. This group is used to classify geometries up to isomorphism, two geometries $(\Gamma, G)$ and $\left(\Gamma^{\prime}, G\right)$ being isomorphic if there exists an element $g$ in $\operatorname{Aut}(G)$ such that $g(\Gamma)=\Gamma^{\prime}$.

Let $C_{1}$ be a set of sets containing one representative of each conjugacy class of maximal subgroups of $G$. Each element $\left\{G_{1}\right\}$ of $C_{1}$ (where $G_{1}$ is thus a maximal subgroup of $G$ ) gives a rank one geometry $\Gamma\left(G,\left\{G_{1}\right\}\right)$.

If $C_{1}$ is nonempty, let $C_{2}$ be the empty set and do the following:

For every set $\left\{G_{1}\right\}$ in $C_{1}$, determine, up to isomorphism, the subgroups $G_{2}$ in $S$ such that $\Gamma\left(G ; G_{1}, G_{2}\right)$ is firm, residually connected and flag-transitive ${ }^{1}$. Store these pairs $\left\{G_{1}, G_{2}\right\}$ in $C_{2}$.

If $C_{2}$ is nonempty, let $C_{3}$ be the empty set and do the following: for every element $\left\{G_{1}, G_{2}\right\}$ in $C_{2}$, determine, up to isomorphism, the subgroups $G_{3}$ in $S$ such that $\Gamma(G$; $\left.G_{1}, G_{2}, G_{3}\right)$ is firm, residually connected and flag-transitive. Store these triples $\left\{G_{1}, G_{2}\right.$, $\left.G_{3}\right\}$ in $C_{3}$.

And so on, until the set $C_{i+1}$ obtained by adding subgroups to elements of $C_{i}$ is empty. The sets $C_{j}, j=1, \ldots, i$, contain all firm, residually connected, flag-transitive and primitive geometries of rank $j$ of $G$, up to isomorphism.

In the algorithm above, the bottleneck is the construction of the group $W$ at the very beginning. That task of computing all maximal subgroups of $G$ becomes quickly tedious

\footnotetext{
${ }^{1}$ In the rank two case, every possible $G_{2} \neq G_{1}$ is good, and the group $W$ is there to make sure we pick only pairwise non-isomorphic pairs of subgroups $\left(G_{1}, G_{2}\right)$.
} 
and the permutation representation of $G$ on these subgroups has a degree that is quickly too large for MAGMA to work efficiently with it.

Given a rank two coset geometry $\Gamma:=\Gamma\left(G ;\left(G_{1}, G_{2}\right)\right)$ where $G_{1}$ and $G_{2}$ are maximal subgroups of $G$, the group $G$ has a faithful primitive permutation representation on the set $\Omega$ of cosets of $G_{1}$ (and respectively on the cosets of $G_{2}$ ). In that permutation representation, $G_{2}$ partitions the cosets into orbits. As $G_{1}$ is maximal in $G$ and the representation is faithful, all the conjugates of $G_{1}$ correspond to stabilizers of one coset of $\Omega$. Hence, by fixing $G_{2}$ and taking stabilizers of representatives of the orbits of $G_{2}$ on $\Omega$, we construct all possible rank two geometries $\Gamma\left(G ;\left(H_{1}, H_{2}\right)\right)$ with $H_{1}$ conjugate to $G_{1}$ and $H_{2}$ conjugate to $G_{2}$. One of them is necessarily the starting geometry.

An algorithm follows from the above paragraph: Given a group $G$ and a maximal subgroup $M_{1}$ of $G$, construct the permutation representation of $G$ on the set $\Omega$ of right cosets of $M_{1}$. This representation is primitive. For any maximal subgroup $M_{2}$ of $G$ in that representation, compute the orbits $\mathcal{O}$ of $M_{2}$ on $\Omega$. In each orbit $o \in \mathcal{O}$, pick one representative $x$. The triple $\left(G ;\left(M_{2}, G_{x}\right)\right)$ gives a primitive geometry of rank 2 . This geometry is obviously flag-transitive and it will be firm provided $|o| \neq 1$. This gives an obvious way to produce primitive geometries of rank 2 for a given group $G$. Any rank two primitive geometry constructed from $G$ can be produced in this way. Therefore, we have a technique to produce all rank two primitive geometries for $G$. It may happen that we generate several isomorphic copies of the same geometry. To avoid that, every time we generate a geometry with the above technique, we check whether it is isomorphic to one of the geometries obtained so far. We only keep it if it is not isomorphic to any of the previously obtained geometries.

In order to be able to apply the above algorithm, all that is needed now is that we can compute permutation representations of $G$ on all its maximal subgroups and that we can check isomorphism between geometries that may be self-dual. Starting from the Rudvalis group, these permutation representations can become very large. This is where we decided to stop.

We implemented the Algorithm 3.1 in MAGMA [1] and determined all rank two primitive geometries for the twelve smallest sporadic simple groups. Our findings are summarized in the next section.

\section{Primitive rank two geometries of sporadic groups}

In this section, we summarise the classifications of primitive geometries of rank two we obtained, using the algorithm described in the previous section, for the five Mathieu groups, the first three Janko groups, the McLaughlin group, the Higman-Sims group, the Held group and the Rudvalis group. A summary of the results obtained is available in Table 1. In the last column, the computing time to classify these geometries is given. The computer used was a workstation with 4 Intel Xeon E5 processors with 6 cores each working at $2.9 \mathrm{GHz}$ and $1 \mathrm{~TB}$ of RAM. We give the orbit tables for the groups $M_{11}, M_{12}, M_{22}, M_{23}$ and $J_{1}$ in the following sections. We omit the tables of the remaining groups since these tables become too large.

\subsection{The Mathieu group $M_{11}$}

Table 2 gives the orbit lengths for every primitive permutation representation of $M_{11}$. Each column corresponds to a given primitive permutation representation and gives the ways 


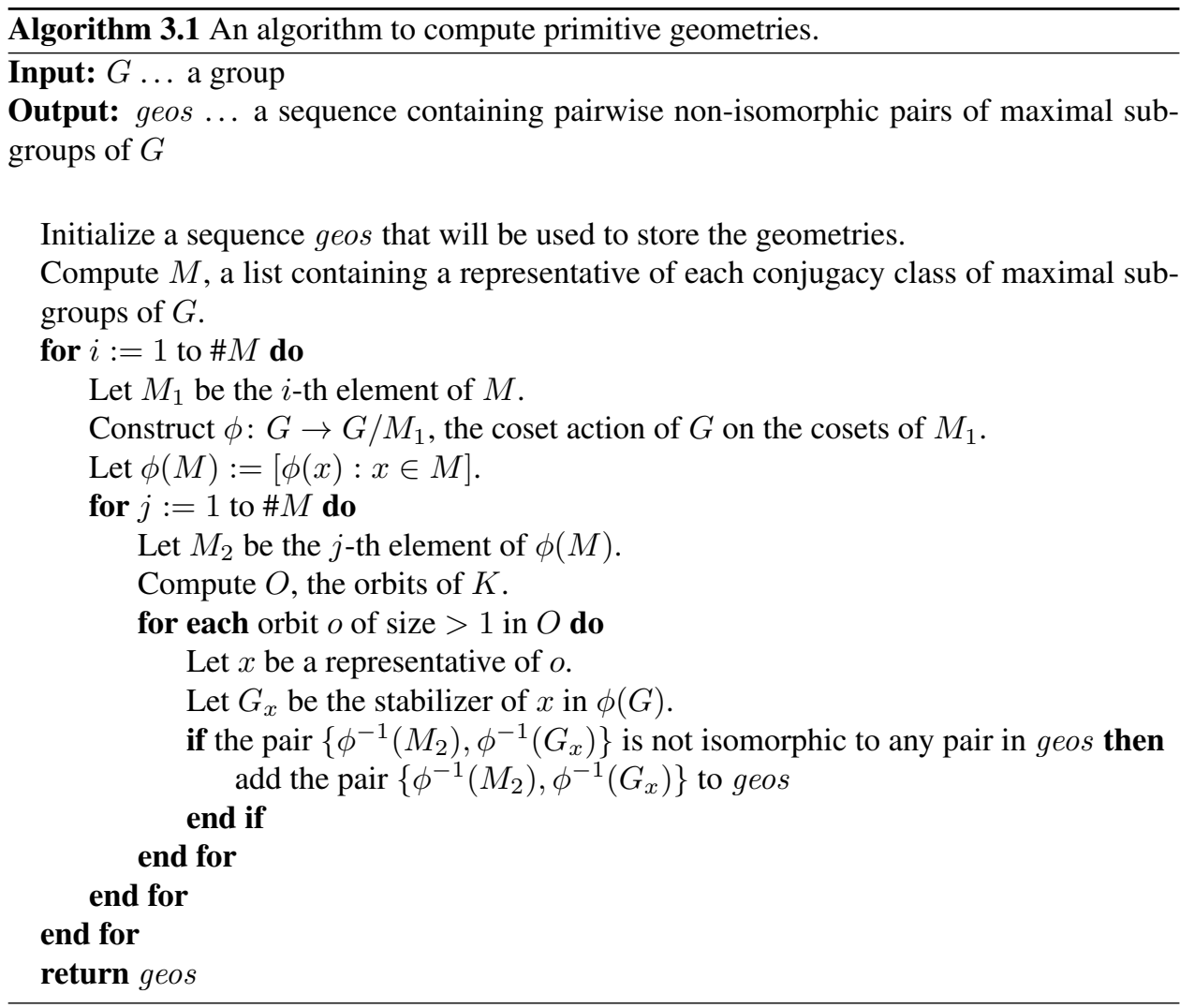

Table 1: The 11 smallest sporadic groups and their rank two primitive geometries.

\begin{tabular}{c|r|r|r|r} 
Group & Order & Degree & Number of geometries & Computing time \\
\hline \hline$M_{11}$ & 7920 & 11 & 37 & $0.28 \mathrm{~s}$ \\
$M_{12}$ & 95040 & 12 & 166 & $13.79 \mathrm{~s}$ \\
$M_{22}$ & 443520 & 22 & 81 & $3.23 \mathrm{~s}$ \\
$M_{23}$ & 10200960 & 23 & 170 & $54.04 \mathrm{~s}$ \\
$M_{24}$ & 244823040 & 24 & 5074 & $106704 \mathrm{~s} \sim 29 \mathrm{~h}$ \\
\hline$J_{1}$ & 175560 & 266 & 669 & $146.22 \mathrm{~s}$ \\
$J_{2}$ & 604800 & 100 & 334 & $71.27 \mathrm{~s}$ \\
$J_{3}$ & 50232960 & 6156 & 546 & $5031.72 \mathrm{~s}$ \\
\hline $\mathrm{HS}$ & 44352000 & 100 & 339 & $282.46 \mathrm{~s}$ \\
$\mathrm{McL}$ & 898128000 & 275 & 443 & $2412.12 \mathrm{~s}$ \\
$\mathrm{He}$ & 4030387200 & 2058 & 4074 & 14.35 days \\
$\mathrm{Ru}$ & 145926144000 & 4060 & 1511 & 9.2 days
\end{tabular}


the points are split into orbits by the corresponding maximal subgroups. So for instance, the entry $12-18-36$ corresponding to the line labelled $M_{9}: 2$ and the column labelled 66 means that on the 66-points primitive permutation representation of $M_{11}$ (obtained by looking at the coset action of $M_{11}$ on the cosets of a maximal subgroup isomorphic to $S_{5}$ ), a maximal subgroup $M_{9}: 2$ has orbits of length 12,18 and 36 .

Counting the number of orbits of size at least two in the upper triangular half, we get 38 possibilities. In fact, the Mathieu group $M_{11}$ has, up to isomorphism, 37 primitive geometries of rank two as two of them are dual of each other. This confirms the results obtained in [5]. In Table 4, we give for each of the 37 geometries $\Gamma$ the designs corresponding to $\Gamma$ and to its dual $\Gamma^{*}$. Some geometries do not appear in that table as a rank two geometry does not necessarily give a design. Entry $28^{*}$ is the well known $S_{1}(4,5,11)$, that is the Steiner system associated to the Mathieu group $M_{11}$.

Table 2: Orbits of primitive permutation representations of $M_{11}$.

\begin{tabular}{c||c|c|c|c|c} 
& 11 & 12 & 55 & 66 & 165 \\
\hline \hline$M_{10}$ & $1-10$ & 12 & $10-45$ & $30-36$ & $45-120$ \\
\hline$L_{2}(11)$ & 11 & $1-11$ & 55 & $11-55$ & $55-110$ \\
\hline$M_{9}: 2$ & $2-9$ & 12 & $1-18-36$ & $12-18-36$ & $9-12-72^{2}$ \\
\hline$S_{5}$ & $5-6$ & $2-10$ & $10-15-30$ & $1-15-20-30$ & $10-15-20-60^{2}$ \\
\hline$M_{8}: S_{3}$ & $3-8$ & $4-8$ & $3-4-24^{2}$ & $4-6-8-24^{2}$ & $1-8-12-24^{4}-48$
\end{tabular}

\subsection{The Mathieu group $M_{12}$}

Table 5 gives the orbit lengths for every primitive permutation representation of $M_{12}$. Counting the number of orbits of size at least two in the upper triangular half, we get 268 possibilities. In fact, the Mathieu group $M_{12}$ has 166 primitive geometries of rank two as $\operatorname{Aut}\left(M_{12}\right)$ conjugates several pairs in the 268 possibilities.

\subsection{The Mathieu group $M_{22}$}

Table 6 gives the orbit lengths for every primitive permutation representation of $M_{22}$. Our programs gave 81 geometries up to isomorphism. This corrects the number that was obtained in [12], namely 86.

\subsection{The Mathieu group $M_{23}$}

Table 7 gives the orbit lengths for every primitive permutation representation of $M_{23}$. Our programs gave 170 geometries up to isomorphism. This confirms the results obtained by Kilic in [10].

\subsection{The first group of Janko $J_{1}$}

Table 8 gives the orbit lengths for every primitive permutation representation of $J_{1}$. Our programs gave 669 geometries up to isomorphism. This is a completely new result. 
Table 3: The 37 rank two primitive geometries of $M_{11}$.

\begin{tabular}{|c|c|c|c|c|}
\hline Nr. & $G_{1}$ & $G_{2}$ & $G_{1} \cap G_{2}$ & {$\left[d_{p}, g, d_{l}\right]$} \\
\hline 1 & $\mathrm{GL}_{2}(3)$ & $\mathrm{GL}_{2}(3)$ & $S_{3}$ & {$[5,3,5]$} \\
\hline 2 & $\mathrm{GL}_{2}(3)$ & $\mathrm{GL}_{2}(3)$ & $E_{4}$ & {$[4,2,4]$} \\
\hline 3 & $\mathrm{GL}_{2}(3)$ & $\mathrm{GL}_{2}(3)$ & $C_{2}$ & {$[4,2,4]$} \\
\hline 4 & $\mathrm{GL}_{2}(3)$ & $\mathrm{GL}_{2}(3)$ & $C_{2}$ & {$[5,2,5]$} \\
\hline 5 & $\mathrm{GL}_{2}(3)$ & $\mathrm{GL}_{2}(3)$ & $C_{2}$ & {$[3,2,4]$} \\
\hline 6 & $\mathrm{GL}_{2}(3)$ & $\mathrm{GL}_{2}(3)$ & 1 & {$[3,2,3]$} \\
\hline 7 & $\mathrm{GL}_{2}(3)$ & $S_{5}$ & $D_{12}$ & {$[5,3,5]$} \\
\hline 8 & $\mathrm{GL}_{2}(3)$ & $S_{5}$ & $D_{8}$ & {$[4,2,4]$} \\
\hline 9 & $\mathrm{GL}_{2}(3)$ & $S_{5}$ & $S_{3}$ & {$[4,2,4]$} \\
\hline 10 & $\mathrm{GL}_{2}(3)$ & $S_{5}$ & $C_{2}$ & {$[3,2,3]$} \\
\hline 11 & $\mathrm{GL}_{2}(3)$ & $S_{5}$ & $C_{2}$ & {$[3,2,3]$} \\
\hline 12 & $\mathrm{GL}_{2}(3)$ & $L_{2}(11)$ & $D_{12}$ & {$[3,2,4]$} \\
\hline 13 & $\mathrm{GL}_{2}(3)$ & $L_{2}(11)$ & $S_{3}$ & {$[3,2,3]$} \\
\hline 14 & $\mathrm{GL}_{2}(3)$ & $M_{9}: 2$ & $Q_{8}: 2$ & {$[6,3,5]$} \\
\hline 15 & $\mathrm{GL}_{2}(3)$ & $M_{9}: 2$ & $D_{12}$ & {$[4,3,4]$} \\
\hline 16 & $\mathrm{GL}_{2}(3)$ & $M_{9}: 2$ & $C_{2}$ & {$[3,2,3]$} \\
\hline 17 & $\mathrm{GL}_{2}(3)$ & $M_{9}: 2$ & $C_{2}$ & {$[3,2,3]$} \\
\hline 18 & $\mathrm{GL}_{2}(3)$ & $M_{10}$ & $Q_{8}: 2$ & {$[3,2,4]$} \\
\hline 19 & $\mathrm{GL}_{2}(3)$ & $M_{10}$ & $S_{3}$ & {$[3,2,3]$} \\
\hline 20 & $S_{5}$ & $S_{5}$ & $D_{8}$ & {$[5,2,5]$} \\
\hline 21 & $S_{5}$ & $S_{5}$ & $S_{3}$ & {$[3,2,3]$} \\
\hline 22 & $S_{5}$ & $S_{5}$ & $E_{4}$ & {$[3,2,3]$} \\
\hline 23 & $S_{5}$ & $L_{2}(11)$ & $A_{5}$ & {$[3,3,4]$} \\
\hline 24 & $S_{5}$ & $L_{2}(11)$ & 12 & {$[3,2,3]$} \\
\hline 25 & $S_{5}$ & $M_{9}: 2$ & 12 & {$[4,2,3]$} \\
\hline 26 & $S_{5}$ & $M_{9}: 2$ & 8 & {$[3,2,3]$} \\
\hline 27 & $S_{5}$ & $M_{9}: 2$ & $C_{4}$ & {$[3,2,3]$} \\
\hline 28 & $S_{5}$ & $M_{10}$ & 24 & {$[3,2,3]$} \\
\hline 29 & $S_{5}$ & $M_{10}$ & 20 & {$[3,2,3]$} \\
\hline 30 & $L_{2}(11)$ & $L_{2}(11)$ & $A_{5}$ & {$[3,2,3]$} \\
\hline 31 & $L_{2}(11)$ & $M_{9}: 2$ & $D_{12}$ & {$[2,2,2]$} \\
\hline 32 & $L_{2}(11)$ & $M_{10}$ & $A_{5}$ & {$[2,2,2]$} \\
\hline 33 & $M_{9}: 2$ & $M_{9}: 2$ & 8 & {$[3,2,3]$} \\
\hline 34 & $M_{9}: 2$ & $M_{9}: 2$ & 4 & {$[3,2,3]$} \\
\hline 35 & $M_{9}: 2$ & $M_{10}$ & $M_{9}$ & {$[3,3,4]$} \\
\hline 36 & $M_{9}: 2$ & $M_{10}$ & 16 & {$[3,2,3]$} \\
\hline 37 & $M_{10}$ & $M_{10}$ & $M_{9}$ & {$[3,2,3]$} \\
\hline
\end{tabular}


Table 4: Designs $S_{\lambda}(t, k, v)$ from the rank two primitive geometries of $M_{11}$.

\begin{tabular}{c|c} 
Nr. & Design \\
\hline \hline 1 & $S_{8}(1,8,165)$ \\
$1^{*}$ & $S_{8}(1,8,165)$ \\
\hline 2 & $S_{12}(1,12,165)$ \\
$2^{*}$ & $S_{12}(1,12,165)$ \\
\hline 3 & $S_{24}(1,24,165)$ \\
$3^{*}$ & $S_{24}(1,24,165)$ \\
\hline 4 & $S_{24}(1,24,165)$ \\
$4^{*}$ & $S_{24}(1,24,165)$ \\
\hline 5 & $S_{24}(1,24,165)$ \\
$5^{*}$ & $S_{24}(1,24,165)$ \\
\hline 6 & $S_{48}(1,48,165)$ \\
$6^{*}$ & $S_{48}(1,48,165)$ \\
\hline 7 & $S_{10}(1,4,66)$ \\
$7^{*}$ & $S_{4}(1,10,165)$ \\
\hline 8 & $S_{15}(1,6,66)$ \\
$8^{*}$ & $S_{6}(1,15,165)$ \\
\hline 9 & $S_{20}(1,8,66)$ \\
$9^{*}$ & $S_{8}(1,20,165)$ \\
\hline 10 & $S_{60}(1,24,66)$ \\
$10^{*}$ & $S_{24}(1,60,165)$ \\
\hline 11 & $S_{60}(1,24,66)$ \\
$11^{*}$ & $S_{24}(1,60,165)$ \\
\hline 12 & $S_{3}(3,4,12)$ \\
$12^{*}$ & $S_{4}(1,55,165)$ \\
\hline 13 & $S_{42}(3,8,12)$ \\
$13^{*}$ & $S_{8}(1,110,165)$ \\
&
\end{tabular}

\begin{tabular}{l|c} 
Nr. & Design \\
\hline \hline 14 & $S_{3}(1,9,165)$ \\
$14^{*}$ & $S_{9}(1,3,55)$ \\
\hline 15 & $S_{4}(1,12,165)$ \\
$15^{*}$ & $S_{12}(1,4,55)$ \\
\hline 16 & $S_{24}(1,72,165)$ \\
$16^{*}$ & $S_{72}(1,24,55)$ \\
\hline 17 & $S_{24}(1,72,165)$ \\
$17^{*}$ & $S_{72}(1,24,55)$ \\
\hline 18 & $S_{1}(3,3,11)$ \\
$18^{*}$ & $S_{3}(1,45,165)$ \\
\hline 19 & $S_{1}(8,8,11)$ \\
$19^{*}$ & $S_{8}(1,120,165)$ \\
\hline 20 & $S_{15}(1,15,66)$ \\
$20^{*}$ & $S_{15}(1,15,66)$ \\
\hline 21 & $S_{20}(1,20,66)$ \\
$21^{*}$ & $S_{20}(1,20,66)$ \\
\hline 22 & $S_{30}(1,30,66)$ \\
$22^{*}$ & $S_{30}(1,30,66)$ \\
\hline 23 & $S_{1}(2,2,12)$ \\
$23^{*}$ & $S_{2}(1,11,66)$ \\
\hline 24 & $S_{1}(10,10,12)$ \\
$24^{*}$ & $S_{10}(1,55,66)$ \\
\hline 25 & $S_{10}(1,12,66)$ \\
$25^{*}$ & $S_{12}(1,10,55)$ \\
\hline 26 & $S_{15}(1,18,66)$ \\
$26^{*}$ & $S_{18}(1,15,55)$
\end{tabular}

\begin{tabular}{l|c} 
Nr. & Design \\
\hline \hline 27 & $S_{30}(1,36,66)$ \\
$27^{*}$ & $S_{36}(1,30,55)$ \\
\hline 28 & $S_{5}(1,30,66)$ \\
$28^{*}$ & $S_{1}(4,5,11)$ \\
\hline 29 & $S_{6}(1,36,66)$ \\
$29^{*}$ & $S_{3}(4,6,11)$ \\
\hline 30 & $S_{1}(11,11,12)$ \\
$30^{*}$ & $S_{1}(11,11,12)$ \\
\hline 33 & $S_{18}(1,18,55)$ \\
$33^{*}$ & $S_{18}(1,18,55)$ \\
\hline 34 & $S_{36}(1,36,55)$ \\
$34^{*}$ & $S_{36}(1,36,55)$ \\
\hline 35 & $S_{1}(2,2,11)$ \\
$35^{*}$ & $S_{2}(1,10,55)$ \\
\hline 36 & $S_{1}(9,9,11)$ \\
$36^{*}$ & $S_{9}(1,45,55)$ \\
\hline 37 & $S_{1}(10,10,11)$ \\
$37^{*}$ & $S_{1}(10,10,11)$
\end{tabular}



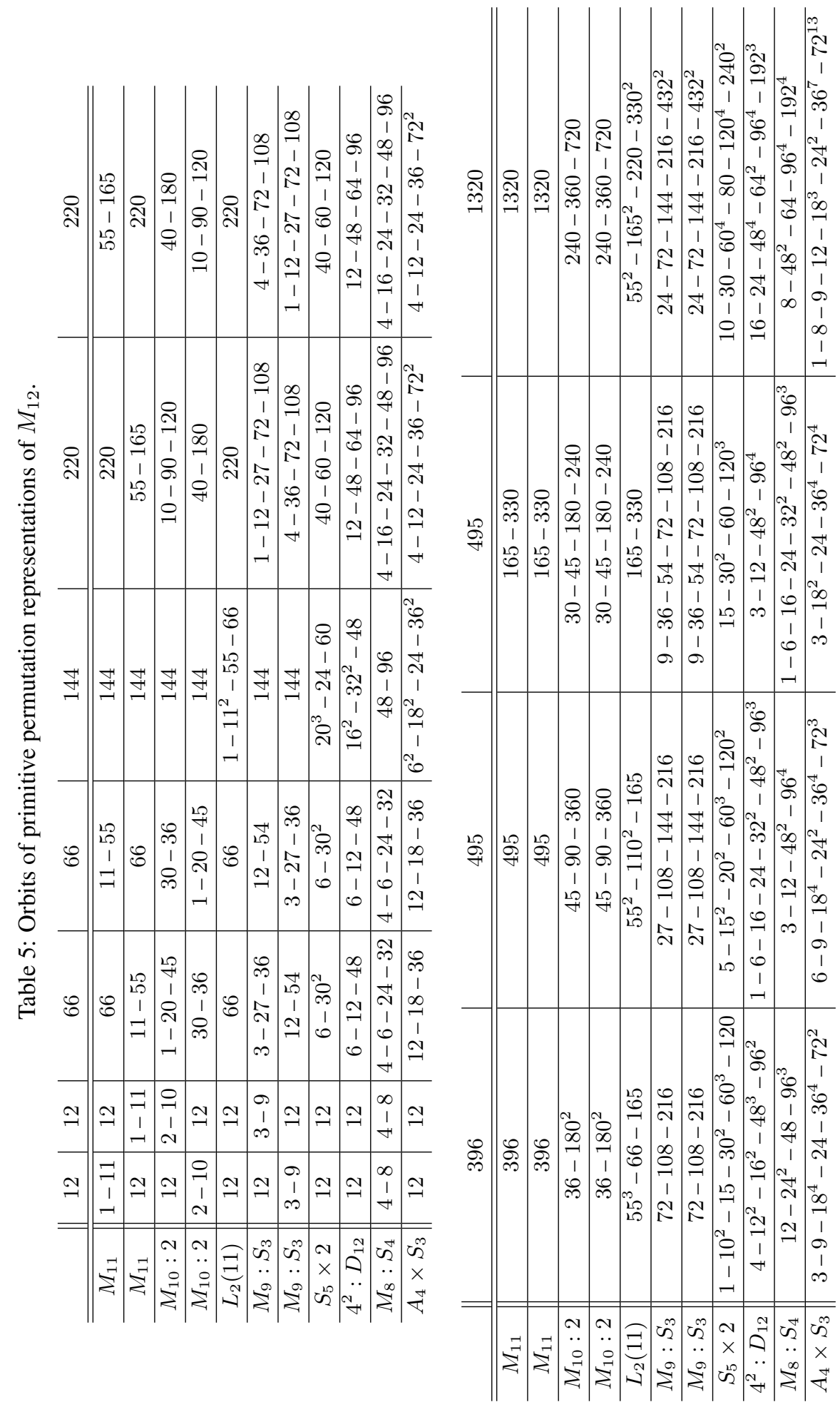
Table 6: Orbits of primitive permutation representations of $M_{22}$.

\begin{tabular}{c||c|c|c|c|c} 
& 22 & 77 & 176 & 176 & 231 \\
\hline \hline$L_{3}(4)$ & $1-21$ & $21-56$ & $56-120$ & $56-120$ & $21-210$ \\
\hline $2^{4}: A_{6}$ & $6-16$ & $1-16-60$ & $80-96$ & $80-96$ & $15-96-120$ \\
\hline$A_{7}$ & $7-15$ & $35-42$ & $1-70-105$ & $15-35-126$ & $21-105^{2}$ \\
\hline$A_{7}$ & $7-15$ & $35-42$ & $15-35-126$ & $1-70-105$ & $21-105^{2}$ \\
\hline $2^{4}: S_{5}$ & $2-20$ & $5-32-40$ & $16-80^{2}$ & $16-80^{2}$ & $1-30-40-160$ \\
\hline $2^{3}: L_{3}(2)$ & $8-14$ & $7-14-56$ & $8-56-112$ & $8-56-112$ & $7-28-84-112$ \\
\hline$M_{10}$ & $10-12$ & $2-30-45$ & $20-36-120$ & $20-36-120$ & $30-36-45-120$ \\
\hline$L_{2}(11)$ & $11^{2}$ & $11^{2}-55$ & $11-55-110$ & $11-55-110$ & $55^{3}-66$
\end{tabular}

\begin{tabular}{c||c|c|c} 
& 330 & 616 & 672 \\
\hline \hline$L_{3}(4)$ & $120-210$ & $280-336$ & $336^{2}$ \\
\hline $2^{4}: A_{6}$ & $30-60-240$ & $16-240-360$ & $96^{2}-480$ \\
\hline$A_{7}$ & $15-105-210$ & $70-126-420$ & $42-210-420$ \\
\hline$A_{7}$ & $15-105-210$ & $70-126-420$ & $42-210-420$ \\
\hline $2^{4}: S_{5}$ & $10-40-120-160$ & $80-96-120-320$ & $160^{3}-192$ \\
\hline $2^{3}: L_{3}(2)$ & $1-7-42-112-168$ & $56^{2}-168-336$ & $112^{3}-336$ \\
\hline$M_{10}$ & $30^{2}-90-180$ & $1-30-45-180-360$ & $72-120^{2}-360$ \\
\hline$L_{2}(11)$ & $55^{3}-165$ & $66-110^{2}-330$ & $1-55^{2}-66-165-330$
\end{tabular}

Table 7: Orbits of primitive permutation representations of $M_{23}$.

\begin{tabular}{c||c|c|c|c} 
& 23 & 253 & 253 & 506 \\
\hline \hline$M_{22}$ & $1-22$ & $77-176$ & $22-231$ & $176-330$ \\
\hline$L_{3}(4): 2$ & $7-16$ & $1-112-140$ & $21-112-120$ & $30-140-336$ \\
\hline $2^{4}: A_{7}$ & $2-21$ & $21-112-120$ & $1-42-210$ & $56-210-240$ \\
\hline$A_{8}$ & $8-15$ & $15-70-168$ & $28-105-120$ & $1-15-210-280$ \\
\hline$M_{11}$ & $11-12$ & $22-66-165$ & $55-66-132$ & $66-110-330$ \\
\hline $2^{4}:\left(3 \times A_{5}\right): 2$ & $3-20$ & $5-48-80-120$ & $3-30-60-160$ & $10-16-120^{2}-240$ \\
\hline $23: 11$ & 23 & 253 & 253 & $253^{2}$
\end{tabular}

\begin{tabular}{c||c|c|c} 
& 1288 & 1771 & 40320 \\
\hline \hline$M_{22}$ & $616-672$ & $231-1540$ & 40320 \\
\hline$L_{3}(4): 2$ & $112-336-840$ & $35-336-560-840$ & 40320 \\
\hline $2^{4}: A_{7}$ & $280-336-672$ & $21-210-420-1120$ & 40320 \\
\hline$A_{8}$ & $168-280-840$ & $35-56-420^{2}-840$ & $20160^{2}$ \\
\hline$M_{11}$ & $1-165-330-792$ & $165-220-330-396-660$ & $720-7920^{5}$ \\
\hline $2^{4}:\left(3 \times A_{5}\right): 2$ & $120-160-240-288-480$ & $1-20-60-90-160-480^{3}$ & $5760^{7}$ \\
\hline $23: 11$ & $23-253^{5}$ & $253^{7}$ & $1-23^{4}-253^{159}$
\end{tabular}




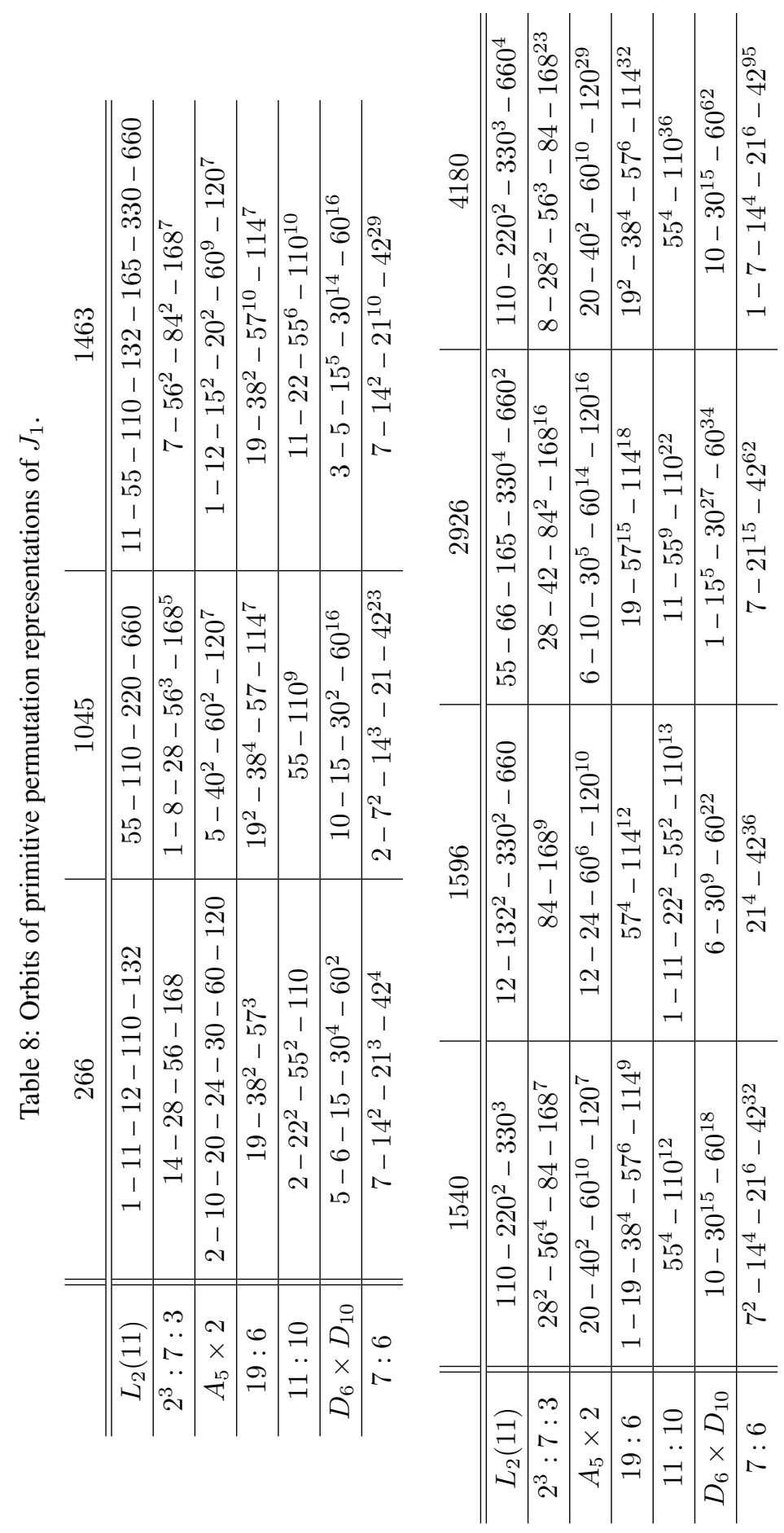




\section{Conclusions}

We have described a new algorithm that allows to compute primitive geometries of rank two for much larger groups than the Dehon algorithm [7]. It remains to extend this algorithm to be able to construct geometries of higher ranks as the Dehon algorithm permitted. Since our first aim was to study codes arising from these rank two geometries, we did not try to extend our algorithm and we leave it as an interesting topic for future work.

The problems found in [12] for $M_{22}$ are very likely due to an incorrect determination of non-isomorphic pairs of subgroups.

\section{References}

[1] W. Bosma, J. Cannon and C. Playoust, The Magma algebra system I: The user language, J. Symbolic Comput. 24 (1997), 235-265, doi:10.1006/jsco.1996.0125.

[2] F. Buekenhout, Diagrams for geometries and groups, J. Comb. Theory Ser. A 27 (1979), 121151, doi:10.1016/0097-3165(79)90041-4.

[3] F. Buekenhout (ed.), Handbook of Incidence Geometry: Buildings and Foundations, NorthHolland, Amsterdam, 1995.

[4] F. Buekenhout, M. Dehon and P. Cara, Geometries of Small Almost Simple Groups Based on Maximal Subgroups, Belgian Mathematical Society, Brussel, 1998, published as a supplement to the Bull. Belg. Math. Soc. Simon Stevin.

[5] F. Buekenhout, M. Dehon and D. Leemans, All geometries of the Mathieu group $M_{11}$ based on maximal subgroups, Experiment. Math. 5 (1996), 101-110, http://projecteuclid. org/euclid.em/1047565641.

[6] M. Dehon, Classifying geometries with CAYLEY, J. Symbolic Comput. 17 (1994), 259-276, doi:10.1006/jsco.1994.1016.

[7] M. Dehon and D. Leemans, Constructing coset geometries with MAGMA: an application to the sporadic groups $M_{12}$ and $J_{1}$, Atti Sem. Mat. Fis. Univ. Modena 50 (2002), 415-427.

[8] N. Kilic, On rank 3 residually connected geometries for $M_{23}$, Int. J. Contemp. Math. Sci. 1 (2006), 679-696, doi:10.12988/ijcms.2006.06071.

[9] N. Kilic, Some rank 3 residually connected geometries for $M_{23}$, Int. J. Contemp. Math. Sci. 1 (2006), 697-718, doi:10.12988/ijcms.2006.06072.

[10] N. Kilic, On rank 2 geometries of the Mathieu group $M_{23}$, Taiwanese J. Math. 14 (2010), 373-387, doi:10.11650/twjm/1500405795.

[11] N. Kilic, On rank 2 geometries of the Mathieu group $M_{24}$, An. Ştiinţ. Univ. "Ovidius" Constanţa Ser. Mat. 18 (2010), 131-147, http://www.anstuocmath.ro/ mathematics/pdf21/13.pdf.

[12] N. Kilic and P. Rowley, On rank 2 and rank 3 residually connected geometries for $M_{22}$, Note Mat. 22 (2003), 107-154, doi:10.1285/115900932v22n1p107.

[13] D. Leemans and B. G. Rodrigues, Binary codes of some strongly regular subgraphs of the McLaughlin graph, Des. Codes Cryptogr. 67 (2013), 93-109, doi:10.1007/s10623-011-9589-7.

[14] J. Tits, Sur les analogues algébriques des groupes semi-simples complexes, in: Colloque d'algèbre supérieure, tenu à Bruxelles du 19 au 22 décembre 1956, Établissements Ceuterick, Louvain, Centre Belge de Recherches Mathématiques, pp. 261-289, 1957. 